\title{
Evaluation of the horizontal load-carrying capacity of a thin steel bridge pier by means of the Damage Subloading Surface model
}

\author{
Fincato Riccardo ${ }^{1, *}$, Tsutsumi Seiichiro ${ }^{1}$, and Momii Hideto $^{2}$ \\ ${ }^{1}$ JWRI, Osaka University, 5670047 11-1 Mihogaoka, Ibaraki, Japan \\ ${ }^{2}$ Nikken Engineering Corporation, 822-0003 4727-1 Kamitonno, Nogata, Fukuoka, Japan
}

\begin{abstract}
The evaluation of the load-carrying capacity of a structure is an important aspect of the structural design. In particular, large seismic events can significantly compromise the performances of structures, or parts of them, due to the formation and accumulation of plastic deformation. In the present paper, we analyse the seismic performance of a thin steel bridge column by means of an unconventional elastoplastic and damage model. The idea is to evaluate the horizontal load-carrying capacity of the pier for different seismic events, analysing the conditions that can compromise the stability of the structure.
\end{abstract}

\section{Introduction}

The evaluation of the capacity of the structure to undergo seismic events, without compromising the stability, is a crucial point for the design. According to international standards, the first step is the identification of the seismic phenomenon in terms of the characterization of the loading wave hitting the structure. One complication in the definition of the load is due to the fact that seismic excitations are quite complex. Their intensity and direction are quite random and it is difficult to define a standard loading wave. Moreover, in most of the cases, the structures are designed to bear the maximum load which reduces the evaluation on one single loading event. One example of experimental work was carried out by Nishikawa et al. [1] after the Kobe earthquake of January 1995, where around $1 \%$ of the steel piers of national roads were significantly damaged. Gao et al. [2] try to model the Nishikawa's experiment using the two-surface model, developed by the Nagoya University, with a good approximation of the inelastic cyclic behaviour of the steel columns. Two loads are applied to the steel pier in both works of Nishikawa et al. and of Gao et al. . The first one is a compressing axial load, which simulates the weight of the superstructure. The second one is cyclic lateral displacement with increasing amplitude through the cycles.

Recently, the authors published a work [3] reproducing the same experimental results carried out by Nishikawa and extending the investigations on nonproportional cyclic loading along two horizontal directions. In [3] the authors adopted an unconventional plasticity model, named Extended Subloading Surface model (i.e. S-S model) [4], with the inclusion of the socalled 'tangential plasticity' $[5,6]$. The numerical results were in good agreement with the real behaviour of the thin wall pier. Moreover, the extension to a bi-directional load offered additional information about the loadcarrying capacity of the structure.

In the present paper, a different constitutive model is adopted, describing the material behaviour within the framework of the Continuum Damage Mechanics (CDM).

An additional internal scalar variable $D$, describing the ductile damage, is coupled with the constitutive equations of the Extended Subloading Surface according to the approach described in [7]. The idea of the present study is to evaluate the structural response under different seismic waves. According to the standard design, the seismic performance in large earthquakes is often evaluated with the maximum loading wave that can hit the structure. However, in most cases, more than one seismic event was registered in the days after the first earthquake, defining the so-called seismic swarm. The application of a series of cyclic loadings, with a smaller amplitude than the one prescribed for the design of the structures, can also jeopardize the stability, due to the plastic strain and damage accumulation.

\section{The Damage S-S model}

\subsection{Constitutive equations}

The Damage Subloading Surface model (i.e. Damage SS) was developed coupling the ductile damage variable with the constitutive equations of the S-S model following the approach proposed by Lemaitre [8]. The constitutive equations are not dealt in this paper, for details the reader is referred to [7], and to [9, 10] for some numerical examples.

The choice of an unconventional plasticity model is due to the ability of the S-S model to catch a realistic accumulation of plastic deformation during cyclic

Corresponding author: fincato@jwri.osaka-u.ac.jp 
loading, as shown in $[11,12]$, or in fatigue life investigations [13, 14, 17]. Briefly, a new surface, i.e. the subloading surface, is obtained from the conventional plastic surface, here named normal-yield surface (see Fig. 1), by means of a similarity transformation. The subloading surface always passes through the current stress state and it contracts or expands, following the loading or unloading of the material. Moreover, the similarity centre is not fixed but it can move in the stress space following the plastic strain rate. This feature allows obtaining close hysteresis loops in the unloadingreloading process [12] with a realistic description of the mechanical ratcheting.

The present work assumes a finite elastoplasticity framework with a hypoelastic based plasticity. The strain rate tensor is decomposed additively into an elastic part $\mathbf{D}^{e}$ and a plastic part $\mathbf{D}^{p}$

$$
\mathbf{D}=\mathbf{D}^{e}+\mathbf{D}^{p}
$$

The coupling between the elasticity and damage is assumed as in [8], through the factor $(1-D)$, that leads to the definition of the corotational rate of the Cauchy stress $\stackrel{\circ}{\sigma}$ as:

$$
\stackrel{\circ}{\boldsymbol{\sigma}}=(1-D) \mathbf{E}: \mathbf{D}^{e}=(1-D) \mathbf{E}:\left(\mathbf{D}-\mathbf{D}^{p}\right)
$$

where $\mathbf{E}$ is the tensor of the elastic constant and $D$ is the isotropic scalar damage variable. The coupling between the plastic strain and the damage is obtained through the consistency condition. The expressions of the normal-yield and subloading surface in the Damage S-S model can be written as:

$$
\begin{gathered}
f(\hat{\boldsymbol{\sigma}})=(1-D) F(H) \\
f(\overline{\boldsymbol{\sigma}})=(1-D) R F(H) \\
\hat{\boldsymbol{\sigma}}=\boldsymbol{\sigma}-\boldsymbol{\alpha}
\end{gathered}
$$

where $f$ is a generic stress function, assumed as the von Mises criterion in this study, $\sigma$ is the Cauchy stress, $\boldsymbol{\alpha}$ is the back stress, $F$ is the isotropic hardening function, $H$ is the isotropic hardening variable (i.e. cumulative plastic strain variable) and $R$ is the similarity ratio. $\bar{\sigma}$ in the second of Eq. (3) represents the Cauchy stress observed by the centre of the subloading surface.

Finally, the expression of the plastic strain rate can be obtained by the material time derivative of the second of Eq. (3) as:

$$
\mathbf{D}^{p}=\frac{\lambda}{(1-D)} \frac{\partial f(\overline{\boldsymbol{\sigma}})}{\partial \boldsymbol{\sigma}}
$$

where $\lambda$ is the plastic multiplier defined as in [7]. Both isotropic and kinematic hardening laws are considered for the material description. The isotropic hardening law is a modified version of the law presented in [15], where an additional linear hardening term is added through the material constant $K$ :

$$
F(H)=F_{0}+K\left(H-H_{d}\right)+F_{0} h_{1}\left[1-e^{h_{2}\left(H-H_{d}\right)}\right]
$$

where $\mathrm{F}_{0}$ is the initial size of the normal-yield surface, $\mathrm{h}_{l}$ and $\mathrm{h}_{2}$ are two material parameters defined by the user and $H_{d}$ is a threshold introduced to simulate the stress plateau. The kinematic hardening law is defined according to the approach suggested by Chaboche [16] with the sum of $N$ non-linear Armstrong-Frederick kinematic laws:

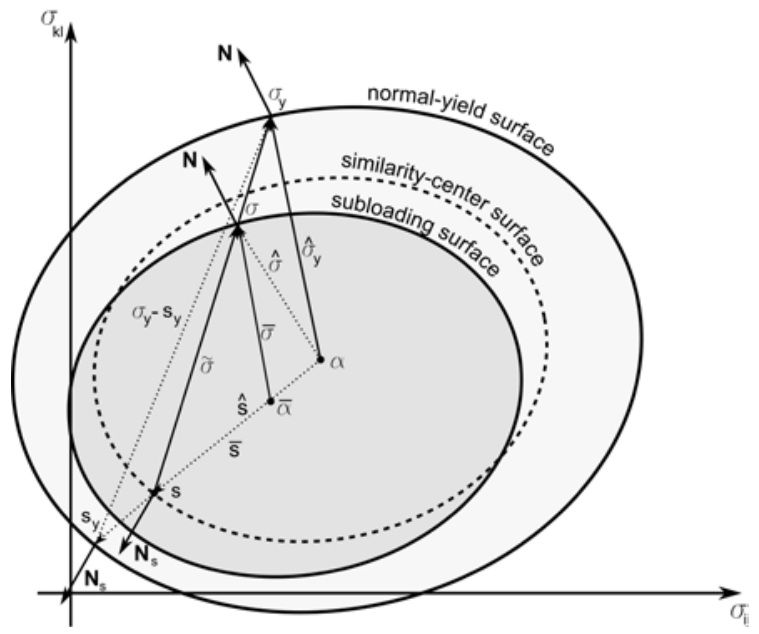

Fig. 1. Sketch of the normal-yield and subloading surface.

$$
\begin{gathered}
\stackrel{\circ}{\boldsymbol{\alpha}}_{i}=\left(C_{i} \mathbf{D}^{p}-\lambda B_{i} \boldsymbol{\alpha}_{i}\right) \\
\stackrel{\circ}{\boldsymbol{\alpha}}=\sum_{i=1}^{n} \stackrel{\circ}{\boldsymbol{o}}_{i}, \quad n=1, \ldots N
\end{gathered}
$$

where $\stackrel{\circ}{\boldsymbol{\alpha}}$ is the corotational rate of the back stress and $C_{i}$, $B_{i}$ are material constants. The definition of the material parameters is discussed in the following section 3.2.

\subsection{Ductile damage criteria}

It is well established that the mechanism that leads to the progressive failure of the metallic materials is due to the presence of impurities in the media. The application of external solicitations causes the formation of micro voids around the impurities, their growth and coalescence until the formation of a crack. Nowadays, in the panorama of the CDM, several theories try to reproduce this phenomenon with more or less complicated models. One common aspect is represented by the individuation of the key factors that affect the damage evolution: the stress triaxiality and the Lode angle.

Previous works of the authors [7,9] implemented the original Lemaitre's damage evolution law, which can consider the exponential decay of the ductility with the increase of the stress triaxiality but cannot describe the dependency of the damage evolution to the different loading conditions. An attempt to include the Lode angle effect on the Lemaitre's law was carried out in [10], where the authors reproduced the failure behaviour of Q460 steel round notched bars and flat grooved plates [18].

Recent works of Bai and Wierzbicki [19] and Algarni et al. [20, 21] implements a modified MohrCoulomb (M-C) criterion for the ductile fracture. This failure criterion has been extensively used for the description of porous media, such as rocks and soils, however, its ability to consider the stress triaxiality $\eta$ and Lode angle parameter $\bar{\theta}$ effects makes it suitable 
for the description of metallic materials too. The definition of the equivalent strain to fracture can be, in fact, expressed as a function of these two parameters only, which are analytically defined as:

$$
\begin{gathered}
\sigma_{m}=-\frac{1}{3}\left(\sigma_{1}+\sigma_{2}+\sigma_{3}\right) \\
\eta=\sigma_{m} / \sigma_{\text {Mises }} \\
\bar{\theta}=1-\frac{6 \theta}{\pi}
\end{gathered}
$$

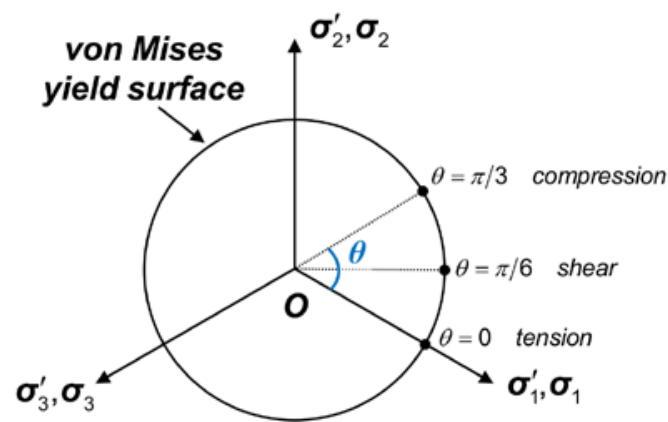

Fig. 2. Schematic representation of a generic stress state in the principal stress space.

where $\sigma_{1}, \sigma_{2}, \sigma_{3}$ are the principal stresses $\left(\sigma_{1} \geq \sigma_{2} \geq \sigma_{3}\right), \sigma_{m}$ is the mean stress, $\sigma_{\text {Mises }}$ is the von Mises stress and $\theta$ is the Lode angle as reported in Fig. 2.

The set of admissible values for the stress triaxiality and the Lode angle parameter and the equivalent strain to fracture defines the 'fracture envelope' $\bar{\varepsilon}_{f}$. Bai and Wierzbicki [9] defined $\bar{\varepsilon}_{f}$ analytically as:

$$
\begin{aligned}
\bar{\varepsilon}_{f}= & \left\{\frac { A } { c _ { 2 } } \left[\sqrt{\frac{1+c_{1}^{2}}{3}} \cos \left(\frac{\bar{\theta} \pi}{6}\right)\right.\right. \\
& \left.\left.+c_{1}\left(\eta+\frac{1}{3} \sin \left(\frac{\bar{\theta} \pi}{6}\right)\right)\right]\right\}^{-\frac{1}{N}}
\end{aligned}
$$

where $A, c_{1}, c_{2}, N$ are four material constants that have to be defined through an experimental campaign. In detail, the formula reported in Eq. (8) is valid uniquely if a von Mises potential is used, otherwise, the general expression requires the calibration of other four material parameters.

This simplification gives a realistic approximation of the ductile behaviour whenever the Lode angle effect on plasticity is limited and the effect of the pressure dependency on the yield surface can be neglected [19]. An application of the $\mathrm{M}-\mathrm{C}$ fracture criterion with a von Mises plastic potential can be found in [22]. Based on Eq. (8) the ductile damage increment can be written as:

$$
\begin{gathered}
\dot{D}=\frac{\sqrt{2 / 3}\left|\mathbf{D}^{p}\right|}{\bar{\varepsilon}_{f}(\eta, \bar{\theta})} \mathrm{H}\left(H-d_{1}\right) \\
\mathrm{H}\left(H-d_{1}\right)=\left\{\begin{array}{cc}
0, & H-d_{1}<0 \\
1, & H-d_{1} \geq 0
\end{array}\right.
\end{gathered}
$$

Where $d_{l}$ is an additional material parameter and $\mathrm{H}$ represent the Heaviside step function, which was introduced by the authors to allow the damage to evolve whenever the cumulative plastic strain exceeds the parameter $d_{l}$.

\section{Numerical analyses}

The constitutive equations discussed in the previous section 2 were implemented via user subroutine for the commercial code Abaqus (ver. 6.14-5). The calibration of the material parameters for the Damage S-S model was carried out reproducing the experimental results in [1], analysing the horizontal load-carrying capacity of a steel bridge pier subjected to a constant axial compressing load and a cyclic horizontal solicitation. Nishikawa et al. investigated the seismic response of nine steel columns, characterized by different materials and geometry. The analyses reported in this work refer to the sample number 8 in [1], see Fig. 3 and Table 1.

The parameters $\mathrm{H}_{\mathrm{y}}$ and $\delta_{\mathrm{y}}$ in Table 1 represent the horizontal load and horizontal displacement when the specimen yields close to the base (see Fig. 4) and $\mathrm{P}_{\mathrm{y}}$ is the squash load. The values of the geometrical and structural parameters were taken directly from [1].

Table 1 Structural parameters of the specimen.

\begin{tabular}{|c|c|}
\hline $\mathrm{h}[\mathrm{mm}]$ & 3,403 \\
\hline $\mathrm{t}[\mathrm{mm}]$ & 8.70 \\
\hline $\mathrm{D}[\mathrm{mm}]$ & 891.3 \\
\hline $\mathrm{P} / \mathrm{P}_{\mathrm{y}}$ & 0.124 \\
\hline $\mathrm{H}_{\mathrm{y}}[\mathrm{kN}]$ & 414.9 \\
\hline$\delta_{\mathrm{y}}[\mathrm{mm}]$ & 10.5 \\
\hline
\end{tabular}



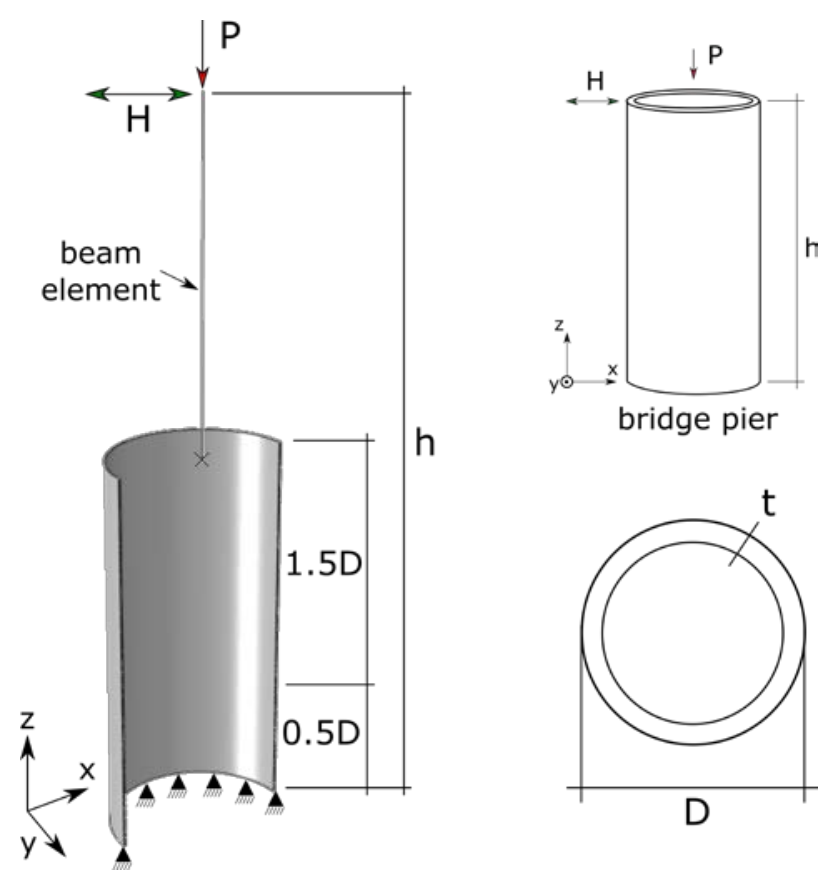

Fig. 3. Model and geometry of the bridge pier.

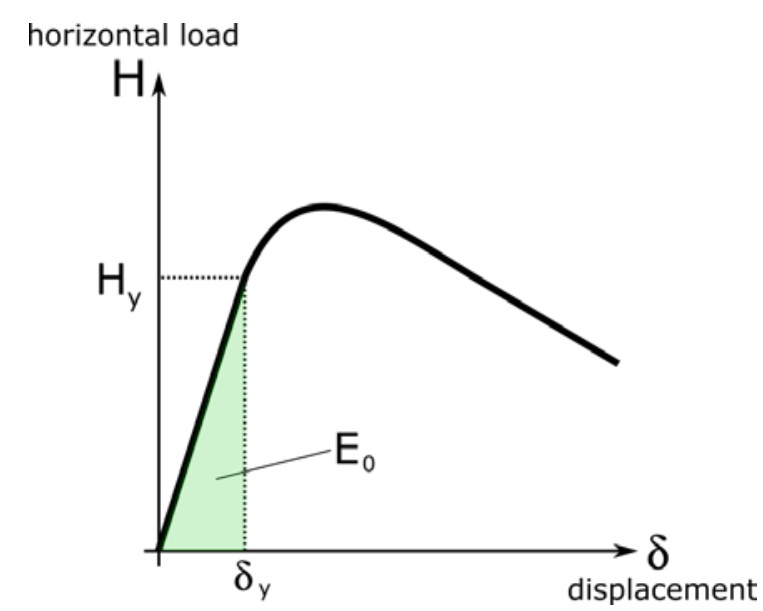

Fig. 4. Lateral earthquake load-displacement relationship.

\subsection{Description of the FE model}

The experimental results in [1] revealed that for thinwalled steel columns of uniform pipe sections, subjected to constant axial compressive load and cyclic lateral displacements, the local buckling always occurs near the base of the columns. Therefore the specimen was modelled using the real circular section from the base of the column to a height equal to two times the external diameter and with a rigid beam for the remaining part, see Fig.3. A similar strategy was also adopted in [2]. Moreover, in order to reduce the computation effort, only half of the column was considered, applying symmetric boundary conditions. The lower part of the pier was meshed with eight-node hexahedral elements (i.e. Abaqus C3D8 elements) and the nodes of the top cross section were connected to the beam with rigid links. A mesh refinement was considered close to the base of the pier, where the maximum accumulation of plastic deformation is expected. The minimum element size is $9.8 \mathrm{~mm}$ (circumference) $\times 2.25 \mathrm{~mm}$ (thickness) $\times$ $15 \mathrm{~mm}$ (axial), comparable with the minimum element size used by Van Do et al. [24], who conducted a mesh size sensitivity analysis on the same study case. The total number of elements amount to 51841 .

The steel pier is compressed with a constant axial load P, simulating the weight of the superstructure. The compressive load is applied at the beginning of the analysis and kept constant during the whole simulation. Four different kinds of horizontal cyclic loads were applied. The first one reproduced the experimental cyclic loading in [1] (i.e. $L 0$ in Fig. 5), the other three simulate the effect of more than one seismic event in sequence. The details about these last three cyclic loading conditions are discussed in section number 3.3.

\subsection{Calibration of the Damage S-S model}

The Damage S-S model requires the calibration of 13 material constants for the elastoplastic behaviour and 4 parameters for the ductile damage behaviour.

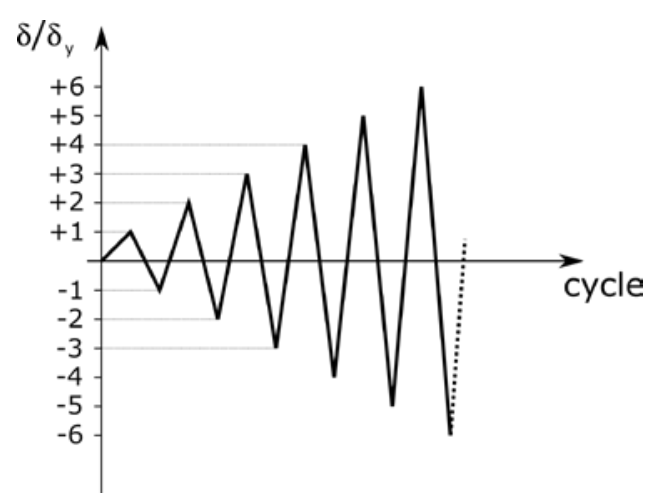

Fig. 5. Loading sequence for the model calibration $(L O)$.

The Young's modulus, the Poisson's ratio and the yield stress were assumed directly from the Nishikawa et al.'s paper, considering a mild SS400 structural steel. The isotropic and kinematic hardening constants of Eqs. (5) and (6) and the 4 material constants for the ductile damage evolution were obtained minimizing the differences between the experimental results in [1] (sample n.8) and the numerical curve obtained with the present model. The values of the elastoplastic constants are reported in the following Table 2, where $R_{e}, u, c$ and $\chi$ are four constants proper of the subloading surface model, the details are available in $[4,25]$. Fig. 6 and Fig. 7 report graphically the calibration of the Damage S-S model for the steel column. As it can be seen the numerical simulation is in good agreement with the experimental results, catching well the normalized horizontal load-normalized horizontal displacement behaviour. 


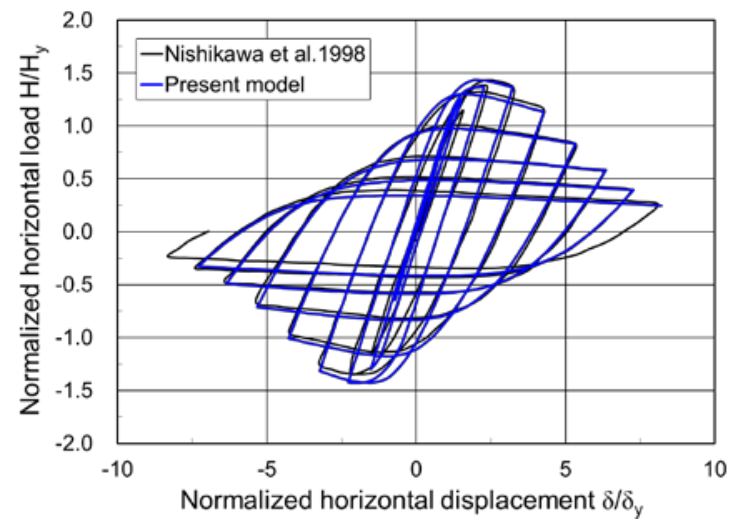

Fig. 6. Hysteresis loops for the pier in Fig. 3 (present model).

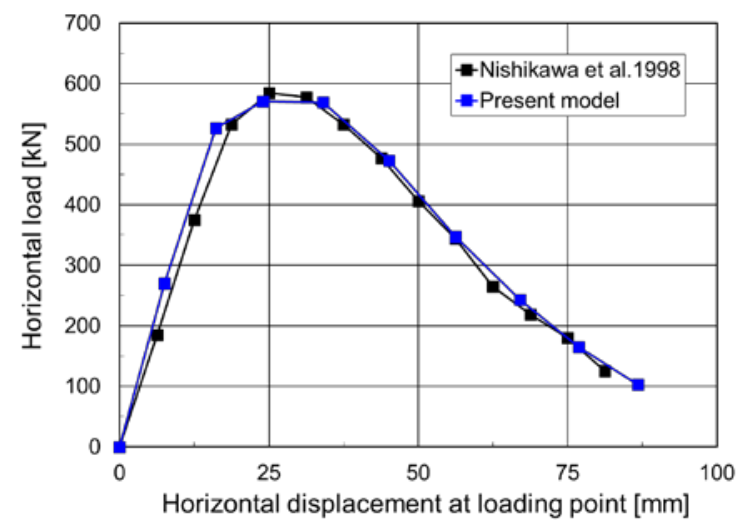

Fig. 7. Envelope of the positive side.

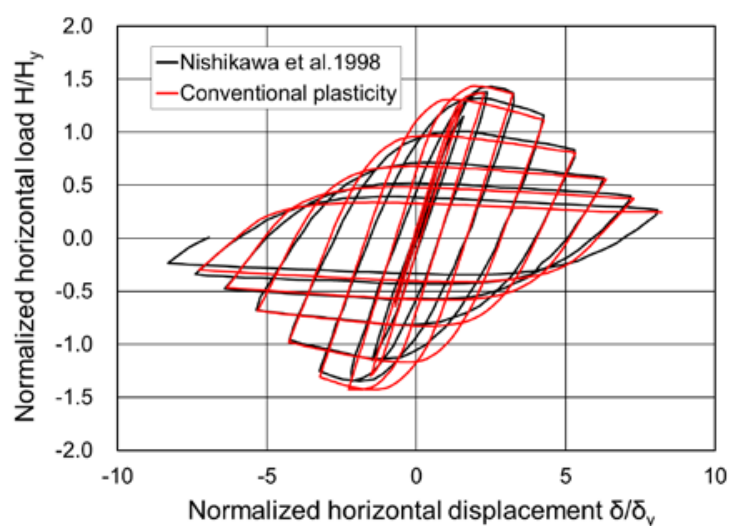

Fig. 8. Hysteresis loops for the pier in Fig. 3 (conventional plasticity).

Moreover, Fig. 7 displays the envelope (positive side) of the horizontal load - horizontal displacement at loading. The load peak value and its horizontal displacement are caught quite well by the model. There is a slight overestimation of the material stiffness in the first part of the curve, probably due to the simplifications and assumptions of the FE model and uncertainties on the parameters. Overall the degree of approximation is satisfactory. Fig. 8 shows the response obtained using a conventional elastoplastic and damage model. In this case, the response of the model seems to be excessively stiff, overestimating the real material performance.

As an additional check, the SS400 steel material parameters in Table 2 were adopted for a uniaxial monotonic extension on a cubic element (C3D8R element). The true stress-cumulative plastic strain is shown in Fig. 9. The value of the $H_{d}$, for the stress plateau, was assumed accordingly to [23]. The present model seems to overestimate the material performances compared with the uniaxial stress-cumulative plastic strain reported in Van Do et al. [24], but they seem to be in good agreement with the curves reported in [23].

The absorbed energy per loop by the steel pier can be represented by the area contained inside the hysteresis loop as schematically depicted in Fig. 10. Fig. 11 reports the comparison of the absorbed energy per loop in [1] against the absorbed energy obtained in the FE simulation, normalized against the elastic energy $E_{0}$. The value of the elastic energy is simply given by the work of the horizontal load at yield $H_{y}$ on the displacement $\delta_{\mathrm{y}}$ (see Fig. 4):

$$
E_{0}=\left(H_{y} \delta_{y}\right) / 2
$$

The response obtained with a conventional elastoplastic model overestimate the absorbed energy in the first six loops. This is due to the fact that the material response during cycles is considered as purely elastic until the stress state reached the plastic potential, generating an overestimation of the hysteresis loops area visible in the previous Fig. 8. Moreover, the normalized absorbed energy of the loop five is almost the same as the one of the loop six anticipating the peak of the absorption for the pier.

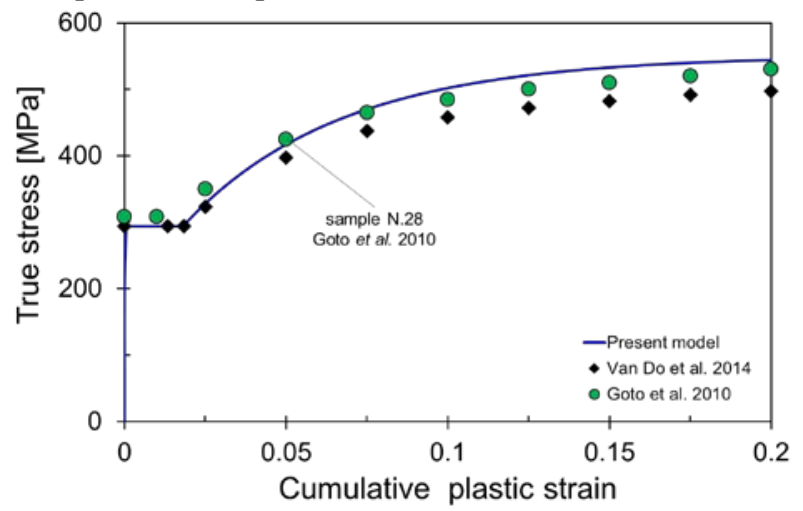

Fig. 9. Uniaxial behaviour of the SS400 steel.

Table 2 Damage S-S parameters.

\begin{tabular}{|c|c|}
\hline Young's modulus $[\mathrm{MPa}]$ & 206000 \\
\hline Poisson's ratio & 0.3 \\
\hline $\mathrm{F}_{0}[\mathrm{MPa}]$ & 294 \\
\hline $\mathrm{u}, \mathrm{c}, \chi$ & $500,200,0.9$ \\
\hline $\mathrm{h}_{1}, \mathrm{~h}_{2}$ & $0.36 ; 15.0$ \\
\hline$K[\mathrm{MPa}]$ & 75 \\
\hline $\mathrm{C}_{1}[\mathrm{MPa}], \mathrm{B}_{1}$ & $3000 ; 23.01$ \\
\hline $\mathrm{C}_{2}[\mathrm{MPa}], \mathrm{B}_{2}$ & $588.6 ; 22.84$ \\
\hline $\mathrm{H}_{\mathrm{d}}$ & 0.183 \\
\hline
\end{tabular}




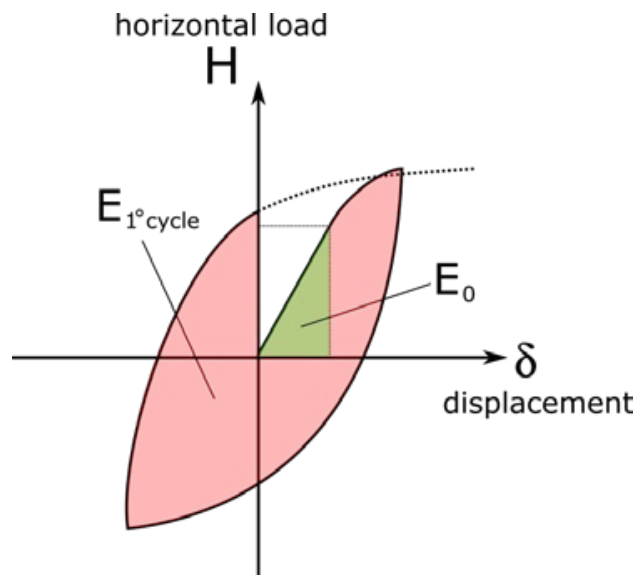

Fig. 10. Example of the absorbed energy during the first cycle.

The overlap between the curves in Fig. 11, for the Damage S-S model, is quite good, with a slight underestimation of the absorbed energy after the sixth cycle. The energy absorption keeps increasing after the peak load is reached (around $\delta=25 \mathrm{~mm}$, see Fig. 7) and reaches it maximum during the sixth cycle. This point can be considered as the limit state from the standpoint of energy absorption capacity for the structure and it can be used to evaluate the seismic performance of the bridge columns.

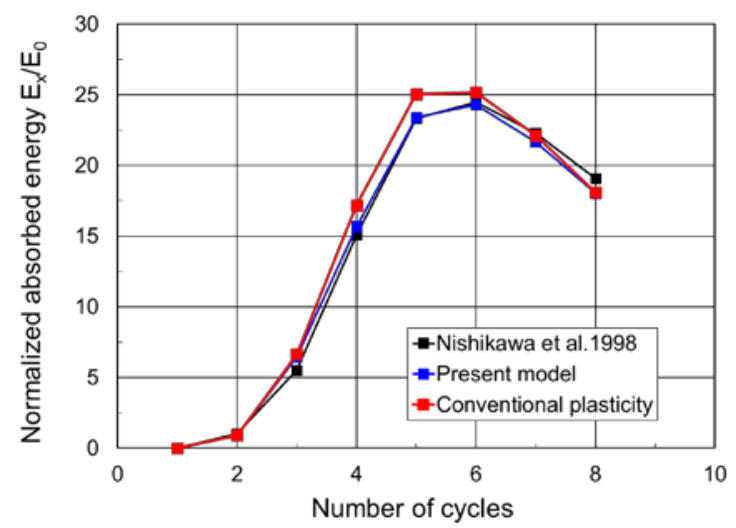

Fig. 11. Energy absorption for the loading sequence of Fig. 5.

The characterization of the M-C criterion for the ductile behaviour of SS400 steel is still missing, therefore in this study the four constants for the definition of the equivalent strain to fracture in Eq. (8) were obtained by fitting the experimental values of the hysteresis loops in Fig. 6. The damage parameters are reported in the following Table 3. In order to make the simulation more realistic, the constants were calibrated starting from the reference values obtained in a different work of the authors (currently under submission [26]) for SM490Y steel. The value of $d_{l}$ was set to $2.5 \%$ in order to simulate the real behaviour of steels which don't show any mechanical degradation under low plastic deformation. Moreover, a critical value for the damage $D_{c}$ was set equal to 0.15 , considering that value as the starting point for the crack opening. The critical damage parameter is included in the present formulation in order to avoid pathological mesh dependency which is the result of the so-called local continuum assumption [27], typical of many models within the CDM.

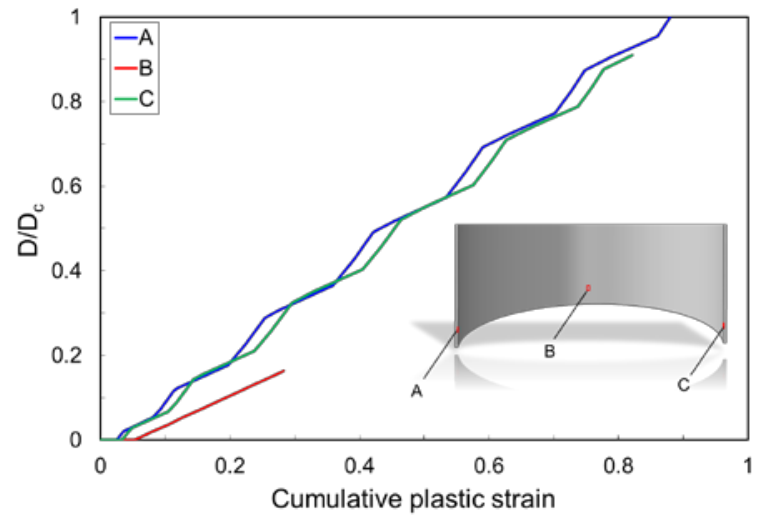

Fig. 12. Damage evolution (element centroid) for three points at the base of the steel pier.

Table $3 \mathrm{M}-\mathrm{C}$ parameters.

\begin{tabular}{|c|c|}
\hline $\mathrm{A}[\mathrm{MPa}]$ & 650 \\
\hline $\mathrm{N}$ & 0.25 \\
\hline $\mathrm{c}_{1}$ & 0.15 \\
\hline $\mathrm{c}_{2}[\mathrm{MPa}]$ & 540 \\
\hline
\end{tabular}

Fig. 12 reports the damage evolution against the cumulative plastic strain at the centroid of three elements located around the base of the steel column, where the local buckling takes place. As expected, the elements A and $\mathrm{C}$ show a higher damage accumulation due to the fact that they are aligned along the loading direction $x$. The damage evolution shows a clear difference in accumulation during the compressive and tensile loading of the pier, due to the asymmetric shape of the fracture envelope (see Fig. 13).

The damage concentrate around the base of the column, where the plastic deformation accumulates. Moreover, the damage contour field shows that the accumulation is higher on the internal wall of the pier rather than on the external wall. The following Fig. 14 and Fig. 15 show the evolution of the stress triaxiality and of the Lode angle parameter for the element A. The dashed blue lines are representative of the average values that are computed using the integral form reported by Bai and Wierzbicki in [9].

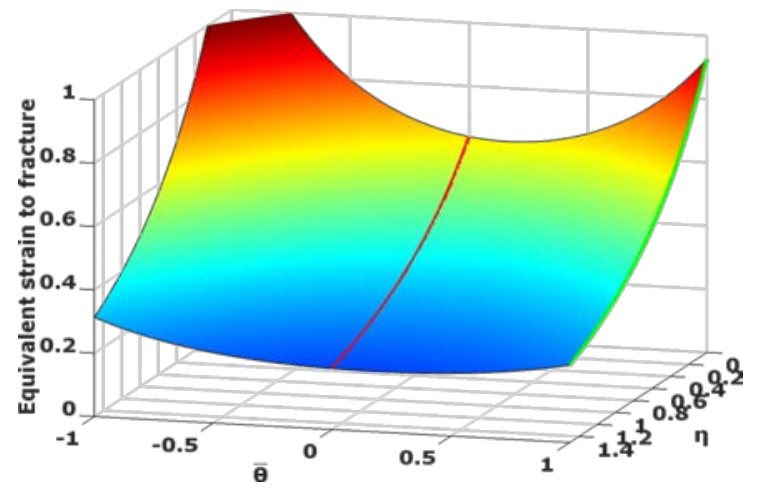


Fig. 13. $\mathrm{M}-\mathrm{C}$ failure envelope.

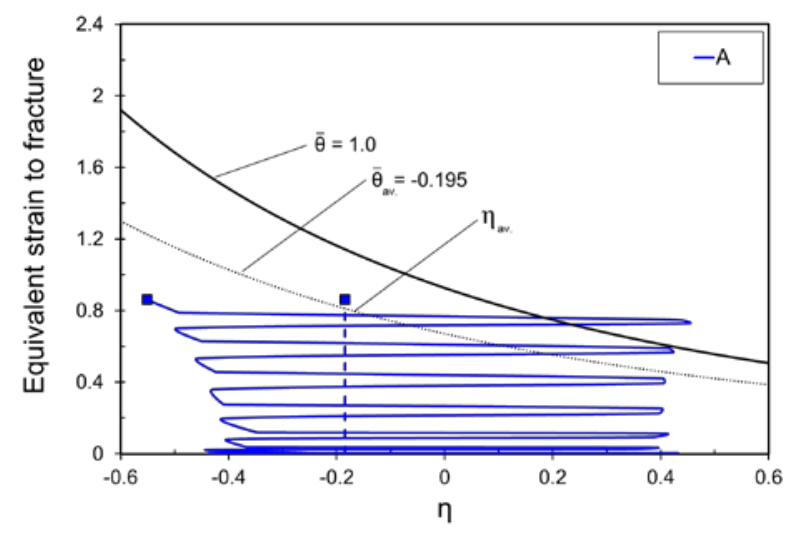

Fig. 14. Stress triaxiality evolution (and its average value) for the element A of Fig. 12 (centroid).

\subsection{Evaluation of the horizontal load-carrying capacity}

The graph of Fig. 11 gave a criterion that allows judging the capability of the structure to absorb energy. The work done by the horizontal solicitation increases until it reaches its peak in the sixth cycle. After that point, the structure is not able to absorb more energy and the area inside the hysteresis loops becomes gradually smaller.

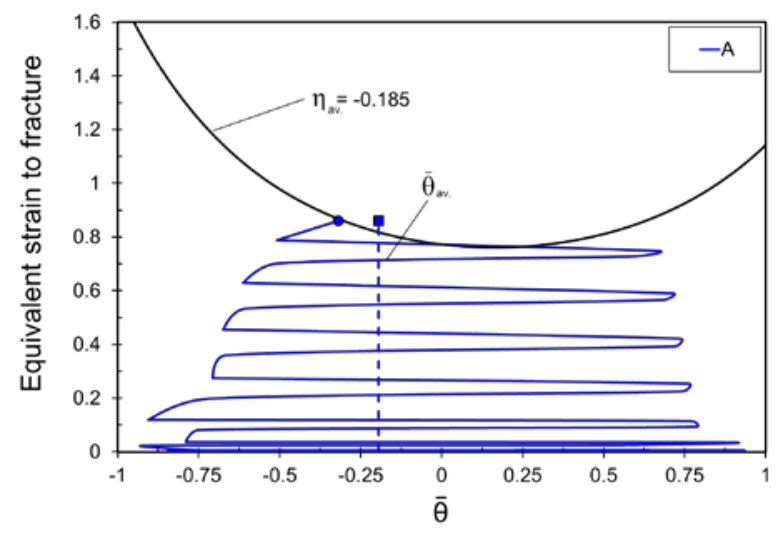

Fig. 15. Lode angle parameter evolution (and its average value) for the element A of Fig. 12 (centroid).

The idea, therefore, is to consider the structure compromised after the sixth cycle.

In reality, earthquakes are characterized by a series of wave loads that take place in a window of time of few days after the first seismic event. In general, the first shock wave is the most intense but often the aftershock waves have an almost similar magnitude (i.e. the earthquake in Tōhoku in 2011 or the central Italy earthquake in 2016). The following Fig. 16 displays the three different loading conditions used to evaluate the horizontal load-carrying capacity of the steel column subjected to a series of shock waves with smaller intensity compared to the one in Fig. 5. Instead of using a monotonically increasing lateral displacement (i.e. $L 0$ loading condition), the three new loading conditions are represented by a sort of harmonic loading, composed by a series of shock waves with different maximum lateral displacements (i.e. $5 \delta_{\mathrm{y}}, 4 \delta_{\mathrm{y}}, 3 \delta_{\mathrm{y}}$ respectively).

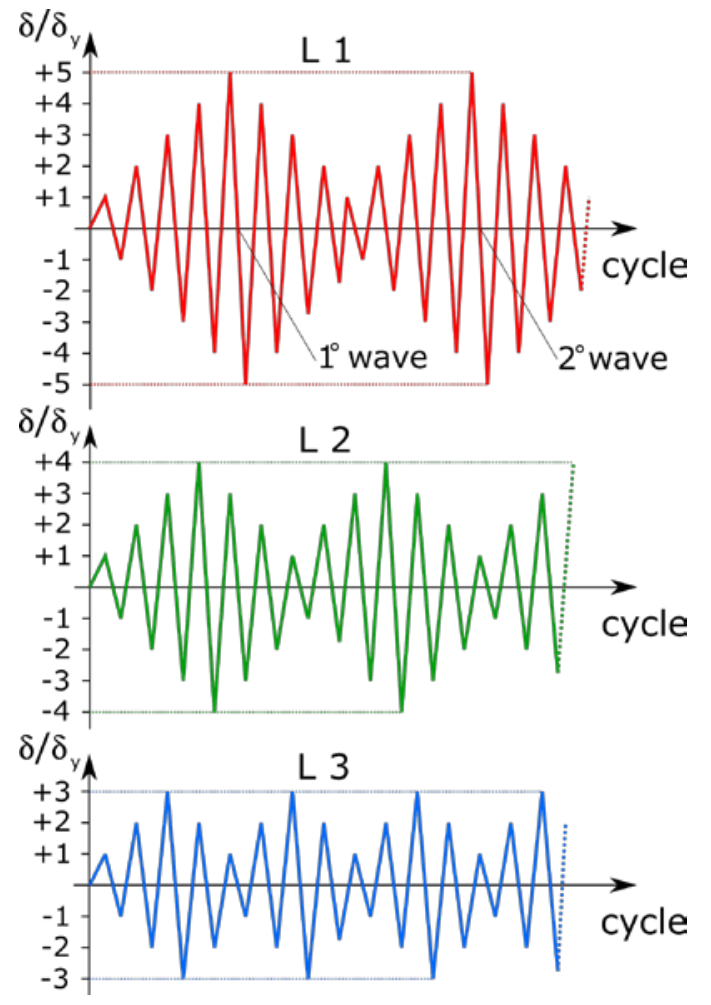

Fig. 16. The three loading sequence for the evaluation of the seismic response of the pier.

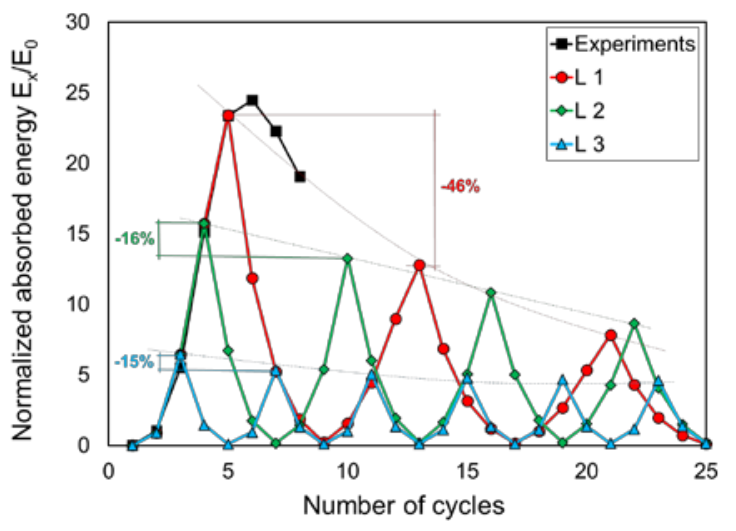

Fig. 17. Absorbed energy per loop for the loading conditions $L$ $1, L 2$ and $L 3$.

Fig. 17 reports the absorbed energy per loop for the three loading conditions of Fig. 16. A maximum number of 25 cycles is displayed to make the graph clearer. The loading condition $L 1$ shows a significant decrease (i.e. $46 \%$ ) in the absorbed energy at the maximum lateral displacement between the first and the second wave. Smaller decreases are instead obtained for the condition $L 1$ and $L 2:-16 \%$ and $15 \%$ respectively. Moreover, the trend for the decrease of the peak seems to be exponential in $L 1$ and almost linear for the other two solicitations.

The definition for the criteria of the load-carrying capacity for the pier is more complicated in case of these new loading conditions since it is not easy to establish a point where the absorbed energy decrease as in Fig. 11. 
However, in a first approximation, it is still possible to establish an energy criteria for the evaluation of the structural performance in terms of cumulative absorbed energy. It is here assumed that the cumulative absorbed energy, in a monotonically increasing loading condition and up to the point when the absorbed energy per loop is maximized, can represent the criteria to evaluate different loading conditions. In other words, the total absorbed energy for the $L 1, L 2$ and $L 3$ loading cases are compared with the total absorbed energy up to the sixth cycle of the monotonically increasing load $E_{\text {crit. }}$.

$$
E_{\text {crit. }}=\sum_{i=1}^{6} E_{i-l o o p}^{L 0}
$$

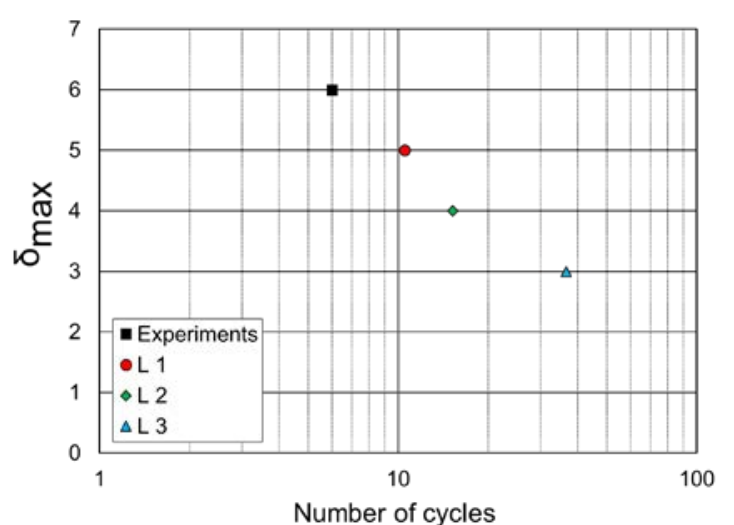

Fig. 18. Number of cycles vs maximum later displacement when the critical value of the total absorbed energy is reached for the $L 1, L 2$ and $L 3$ loading conditions.

Fig. 18 displays the number of cycles when the critical absorbed energy, defined in Eq. (11), is reached for the $L 1, L 2$ and $L 3$ loading conditions. As expected, the number of cycles increases with the decrease of the maximum imposed displacement $\delta_{\max }$. The black, the red and green points are almost aligned, showing a linear increase in the number of cycles. When the loading amplitude becomes smaller the number of cycles sensibly increase, due to the fact that the material tends to behave elastically. It is worth mentioning, at this point, that the energy criteria defined in Eq. (11) is subjected to some uncertainties. First, the ductile damage law defined in Eq. (9) is characterized by a linear damage evolution which overestimates the damage accumulation during cycles. This leads to a smaller absorbed energy per cycle due to the material softening and therefore to an overestimation of the number of cycles to reach the critical energy. Moreover, the ductile damage accumulation law should be also provided with a term that takes into account the non-proportionality of the load, since it has been shown that this factor affects the ductility of metals $[20,28]$. On the other hand, the criteria can provide, in first approximation, a valid tool to judge the seismic performance of steel bridge columns under unidirectional seismic loading.

\section{Conclusions}

In this paper, the performance of a steel bridge columns in terms of horizontal load-carrying capacity was evaluated. The pier behaviour under a constant compressive load and horizontal cycling loading were investigated by means of an unconventional elastoplastic and damage model named Damage S-S model. The results can be summarized in the following points:

- The Damage S-S model can catch a realistic structural response of the pier. The graphs reporting the horizontal load vs the horizontal displacement (Fig. 6), the positive load envelope vs lateral displacements (Fig. 7) and the absorbed energy per loop (Fig. 11) showed good agreement with the experimental data reported in [1]. On the other side, the use of a conventional plasticity model overestimate the material performances.

-The damage is localized at the base of the column and it can provide information on the location of the crack formation.

-A criterion for the load-carrying capacity of the structure is provided in terms of cumulative absorbed energy and it is used to judge the seismic performance of the structure subjected to different harmonic loadings. The results showed an increasing number of cycles for a decrease of the maximum imposed displacement.

Future works will focus on the definition of a new ductile damage accumulation law, more suitable for the description of the ductility behaviour under nonproportional cyclic loading.

\section{References}

1. K. Nishikawa, S. Yamamoto, T. Natori, K. Terao, H. Yasunami and M. Terada, Eng. Struct., 20, 540, (1998)

2. S. Gao, T. Usami, H. Ge, J. Eng. Mech., 124, 260 (1998).

3. H. Momii, S. Tsutsumi, R. Fincato, Transaction of JWRI, 44, 41 (2015)

4. K. Hashiguchi, Elastoplasticity Theory (Springer, Berlin, 2009)

5. K. Hashiguchi, S. Tsutsumi, Int. J. PLasticity 117, 117 (2001)

6. S. Tsutsumi, K. Hashiguchi, Int. J. Plasticity, 21, 1941 (2005)

7. R. Fincato, S. Tsutsumi, Int. J. Num. Meth. Eng. 113, 1729 (2017)

8. J. Lemaitre, J. Engng. Mat. Tech 107, 83 (1985)

9. R. Fincato, S. Tsutsumi, Fracture and Structural Integrity 38, 231 (2016)

10. R. Fincato, S. Tsutsumi, Q. J. Jpn. Weld. Soc. 35, 185s (2017) 
11. K. Hashiguchi, M. Ueno, T. Ozaki, Acta Mech. 223, 985 (2012)

12. R. Fincato, S. Tsutsumi, Marine Struct. 55, 104 (2017)

13. S. Tsutsumi, K. Murakami, K. Gotoh, M. Toyosada, JASNAOE 7, 243 (2008)

14. S. Tsutsumi, M. Toyosada, F. Dunne, Procedia Engineering 2, 1392010.

15. K. Hashiguchi, T. Yoshimaru, Int. J. Plast. 11, 347 (1995)

16. J.L. Chaboche, Int. J. Plast. 2, 149 (1986)

17. S. Tsutsumi, K. Morita, R. Fincato, H. Momii, Fracture and Structural Integrity 38, 240 (2016)

18. W. Li, F. Liao, T. Zhou, H. Askes, J. of Constr. Steel Res. 123, 1 (2016)

19. Y. Bai, T, Wierzbicki, Int. J. Fract 161, 1 (2010)

20. M. Algarni, Y. Choi, Y. Bai, Int. J Fatigue 96, 162 (2017)

21. M. Algarni, Y. Bai, Y. Choi, Eng. Fract. Mech. 147, 140 (2015)

22. S. Tsutsumi, T. Kitamura, R. Fincato, Proceedings of TEAM2017, Osaka, Japan

23. Y. Goto, G.P. Kumar, N. Kawanishi, J. Struct. Eng. 36, 1413 (2010)

24. V.N. Van Do, C.H. Lee, K.H. Chang, Comp. Mech. 53, 1209 (2014)

25. S. Tsutsumi, M. Toyosada, K. Hashiguchi, J. of. App. Mech. JSCE, 9, 455 (2006)

26. S. Tsutsumi, T. Kitamura, R. Fincato, Eng. Fract. Mech. (to be published) (2018).

27. J. Besson, Int. J. Dam. Mech. 19, 3 (2010)

28. J. Papasidero, V. Doquet, D. Mohr, Int. J. Solids Struct 69-70, 459 (2015) 